\title{
META-ANÁLISE PARA ESTIMATIVAS DE CORRELAÇÃO GENÉTICA ENTRE PESOS AO NASCER E DESMAMA DE BOVINOS
}

\author{
Juliana Di Giorgio Giannotti ${ }^{1}$; Irineu Umberto Packer²*; Maria Eugênia Zerlotti Mercadante ${ }^{3}$ \\ ${ }^{1}$ Pós-Graduanda em Ciência Animal e Pastagens - USP/ESALQ. \\ ${ }^{2}$ Depto. de Produção Animal - USP/ESALQ, C.P. 9 - CEP: 13418-900 - Piracicaba, SP. \\ ${ }^{3}$ Instituto de Zootecnia - EEZ Sertãozinho, C.P. 63 - CEP: 14160-000 - Sertãozinho, SP. \\ *Autor correspondente <iupacker@esalq.usp.br>
}

\begin{abstract}
RESUMO: O problema de combinar informações de estudos independentes atinge quase todos os campos da ciência, devido, principalmente, ao grande número de artigos científicos publicados nos últimos anos, assim sumarizar informações se tornou uma necessidade. Uma meta-análise foi conduzida com o objetivo de resumir as estimativas da correlação genética entre pesos ao nascer e desmama de bovinos de corte de origem zebuína. Foi usado um conjunto de estimativas obtidas em 39 artigos publicados no período de 1968 a 2000. Os dados foram analisados de acordo com modelos fixo e aleatório, este último sob a técnica da máxima verossimilhança restrita. O modelo fixo não foi apropriado em virtude da falta de homogeneidade das estimativas de correlação, sendo portanto recomendável o modelo aleatório. A análise de subgrupo, estratificado por década de publicação, também apresentou heterogeneidade. A meta-análise constitui técnica que deve ser implementada para esta e outras finalidades

Palavras-chave: correlação genética, estimativa combinada, revisão quantitativa
\end{abstract}

\section{META-ANALYSIS FOR ESTIMATES OF GENETIC CORRELATION BETWEEN BIRTH AND WEANING WEIGHTS OF CATTLE}

\begin{abstract}
The problem of combining information from independent studies permeates almost all fields of science. Because the number of scientific articles being published has increasingly grown in past years, combining information has became a necessity. A meta-analysis was conducted to summarize published estimates of genetic correlation between birth and weaning weights of zebu beef cattle, through the collection of estimates of 39 reports, from 1968 to 2000. Data were analyzed by fixed and random effect models. Components of variance were obtained by the restricted maximum likelihood technique. Strong lack of homogeneity among the studies was observed. As a consequence, fixed model estimates of combined results were inappropriate. Results suggest that random effect models produce better estimates. Subgroup comparisons, for decade publication, showed heterogeneity. Meta-analysis techniques were recommended for quantitative reviews of genetic parameters.
\end{abstract}

Key words: genetic correlation, pooled estimate, quantitative review

\section{INTRODUÇÃO}

A meta-análise utiliza métodos estatísticos para combinar ou comparar resultados de estudos distintos, mas relacionados (Kirby, 1993). O termo meta-análise foi introduzido por Glass (1976), como "a análise das análises" ou "a análise estatística de uma grande coleção de resultados de estudos individuais, com o propósito de completar as descobertas". A principal motivação para a condução de uma meta-análise está no grande número de estudos similares publicados nas diversas áreas da pesquisa, o que vem gerando interesse no desenvolvimento de métodos para combinar informações provenientes dos mesmos.

A meta-análise permite aumentar o número de observações e o poder estatístico dos testes de hipóteses, avaliar a possibilidade de generalizar conclusões para uma amplitude variada de estudos, examinar a variabilidade entre os ensaios ou estudos, resolver incertezas quando certas conclusões destoam; realizar análise de subgrupo, identificar a necessidade e planejar ensaios ou estudos maiores; além de responder questões que não foram atribuídas de início aos estudos individuais. Apesar de oferecer muitos atrativos ela não deve ser vista como substituta para a análise dos dados originais. Mesmo quando meticulosamente realizada, os resultados de uma metaanálise devem ser interpretados com atenção, principalmente devido ao inevitável vício de publicação e à influência do critério de inclusão (Fagard et al., 1996).

Os principais passos envolvidos na meta-análise são: pesquisa bibliográfica, transformação dos resultados de cada estudo numa métrica comum, verificação da homogeneidade dos resultados, modelagem da variação entre estudos e análise de sensibilidade (Wang \& Bushman, 1999). A natureza diversa dos diferentes estudos, tanto em termos de delineamento como em relação aos métodos empregados na realização de cada 
um deles é a principal dificuldade para combinar os resultados (DerSimonian \& Laird, 1986), e uma das estratégias para solucionar esta questão é a incorporação da variação entre os estudos na análise (DerSimonian \& Laird, 1986; Houwelingen, 1997). Assim, as características dos estudos podem ser consideradas como variáveis fixas ou aleatórias e a diferença entre os dois é a maneira com que a variabilidade dos resultados é tratada (Egger et al., 1997). Nos modelos de efeito fixo assume-se que o tamanho do efeito populacional é um valor fixo, enquanto que nos modelos de efeito aleatório é uma variável aleatoriamente distribuída com média e variância próprias (Wang \& Bushman, 1999).

Apesar das críticas e problemas enfrentados pela meta-análise, evidências indicam que ela é um procedimento estatístico que vem sendo aceito pela comunidade científica, e sua aplicação tem aumentado muito nos vários campos das ciências (Cooper, 1990).

No contexto do melhoramento animal são frequentes as revisões bibliográficas sobre estimativas dos parâmetros genéticos (herdabilidade e correlações genéticas) de populações bovinas como pode ser observado em Mohiuddin (1993), Koots et al. (1994a,b), Mercadante et al. (1995) e Lôbo et al. (2000). Nestes trabalhos os autores têm usado vários critérios para combinar as estimativas individuais. De modo geral tais revisões seguem alguns fundamentos da meta-análise, a saber, pesquisa bibliográfica abrangente e obtenção de uma medida resumida comum dos vários estudos, porém, não utilizam de outros que são imprescindíveis na proposta da meta-análise.

Desta maneira, os objetivos deste trabalho consistem na sistematização da metodologia da metaanálise dirigida para a correlação e sua aplicação ao problema específico de resumir estimativas da correlação genética entre pesos ao nascer e desmama em bovinos de origem indiana.

\section{MATERIAL E MÉTODOS}

Os dados utilizados referem-se às estimativas dos coeficientes de correlação genética $(r)$ entre peso ao nascimento e peso à desmama, de bovinos de corte de origem indiana, provenientes de uma revisão realizada por Mercadante et al. (1995) e atualizada até o ano de 2000. A pesquisa bibliográfica realizada por estes autores foi feita de modo a incluir o máximo possível de estudos sobre o tópico em questão, resultando em 37 estudos, entre os anos de 1968 a 1995. A atualização, de 1996 a 2000, foi feita pesquisando em anais de congressos e simpósios e na base de dados CAB, resultando em apenas dois estudos totalizando portanto, 39 referências (Tabela 1). Tais estudos são provenientes de sete países tropicais, oito raças, com número de observações variando de 193 a 31488 , e foram estratificados em três subgrupos, de acordo com a década de publicação: trabalhos publicados até 1970; publicados em década 80 e os da década 90 , identificados como subgrupos "1", "2" e "3", respectivamente. A estratificação por década é justificada pelo avanço das técnicas de estimação de parâmetros genéticos no decorrer dos anos, aliado à melhoria da quantidade e qualidade dos bancos de dados de desempenho de bovinos, de tal modo que ano de publicação pode refletir a qualidade dos resultados. Outras estratificações poderiam ser feitas, porém fogem ao objetivo deste trabalho.

A fim de obter estimativa combinada da correlação genética entre pesos ao nascer e desmama de bovinos de corte foram considerados apenas os estudos com mais de 500 observações, segundo critério proposto por Koots et al. (1994b), tendo em vista que a precisão da estimativa é função direta do tamanho da amostra.

A meta-análise propriamente dita aplicada ao coeficiente de correlação envolveu as seguintes etapas: obtenção do coeficiente de correlação transformado, teste de homogeneidade entre os estudos, modelagem da variação, escolha do modelo mais apropriado para o conjunto total de estudos, análise de subgrupo e análise

A transformação de coeficiente de correlação genética ( $r$ ) para o valor $z$, proposta por Fisher, citado por Hedges \& Olkin (1985), dado por $z=\frac{1}{2} \ln \frac{1+r}{1-r}$, é recomendada pelo fato do valor $z$ apresentar distribuição aproximadamente normal com variância $\hat{\sigma}^{2}=1 /(n-3)$, onde $\mathrm{n}$ é o número de observações de cada estudo.

Com a finalidade de verificar a amplitude da variação dos coeficientes de correlação ( $r$ ) separadamente para cada estudo, construiu-se um "dot plot", com o procedimento TIMEPLOT (SAS, 1990a). Os limites inferior e superior foram obtidos por:

$$
\zeta_{\text {inf. }}=Z-\frac{C_{\alpha} / 2}{\sqrt{n-3}} \text { e } \zeta_{\text {sup. }}=Z+\frac{C_{\alpha} / 2}{\sqrt{n-3}}
$$

onde $C_{\alpha / 2}$ é o quantil de ordem $100(1-\alpha / 2) \%$ da distribuição normal padronizada.

Existem duas formas de combinar as informações na meta-análise, de acordo com a modelagem da variação entre as diferentes estimativas, a qual pode ser sob os modelos fixo ou aleatório. Para decidir qual é mais apropriada para os dados em questão, efetuou-se um teste de homogeneidade entre os estudos (Costa, 1999), através do procedimento GLM (SAS, 1990b), testando a hipótese, $H_{0}: \theta_{1}=\theta_{2}=\ldots=\theta_{k}$ ( $k$ é o número de estudos) versus $\mathrm{H}_{\mathrm{A}}$ : pelo menos um coeficiente de correlação difere dos demais, através da estatística:

$$
Q_{\text {entre }}=\sum_{i=1}^{k} w_{i+}\left(\hat{\theta}_{i+}-\hat{\theta}_{++}\right)^{2}
$$

a qual tem distribuição de qui-quadrado com $p-1$ graus de liberdade, onde: 
$W_{i+}=\sum_{j=1}^{m_{i}} W_{i j} ; W_{i j}=\frac{1}{\operatorname{Var}\left(\hat{\theta}_{i j}\right)} ; \hat{\theta}_{i+}=\frac{\sum_{j=1}^{m_{i}} W_{i j} \hat{\theta}_{i j}}{\sum_{j=1}^{m_{i}} W_{i j}}$ e $\hat{\theta}_{++}=\frac{\sum_{i=1}^{p} W_{i+} \hat{\theta}_{i+}}{\sum_{i=1}^{p} W_{i+}}$

(Wang \& Bushman, 1999).

Quando se realiza a aproximação por efeito fixo utiliza-se o modelo $y_{i}=\theta+e_{i}$, onde, $y_{i}$ representa os coeficientes de correlação genética transformados, sendo $\mathrm{y}_{\mathrm{i}} \sim \mathrm{N}\left(\theta_{\mathrm{i}}, \sigma_{\mathrm{i}}^{2}\right)$ com $\mathrm{i}=1, \ldots, \mathrm{k} ; \theta$ é a média ponderada de $y_{i}$, dada por $Z_{+}=\frac{\sum_{i=1}^{k} w_{i+} Z_{i}}{\sum_{i=1}^{k} w_{i}}$ a ponderação é $W_{i}=1 / \sigma_{i}^{2} ; e_{i}$ representa o erro aleatório $\sim \mathrm{N}\left(0, \sigma_{\mathrm{i}}^{2}\right)$.

Para a aproximação de efeito aleatório, aplicase o modelo hierárquico, $y_{i}=\theta_{i}+e_{i} ; \theta_{i}=\theta+\varepsilon_{i}$, onde, $y_{i}$ representa os coeficientes de correlação genética

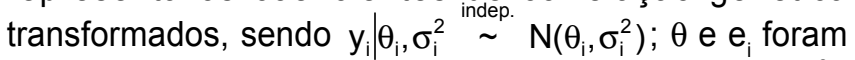
definidos anteriormente, e $\varepsilon_{\mathrm{i}}$, é o erro aleatório $\sim \mathrm{N}\left(0, \tau^{2}\right)$. Neste caso a média ponderada de $\mathrm{y}_{\mathrm{i}}$ é obtida como no modelo fixo, porém, com ponderação $W_{i}^{*}=1 /\left(\sigma_{i}^{2}+\tau^{2}\right) ; \theta_{i}$, é obtido por :

$$
\hat{\theta}_{i}=\left(1-\hat{B}_{i}\right) y_{i}+\hat{B}_{i} \theta_{+} \text {sendo } \hat{B}_{i}=\frac{\hat{\sigma}_{i}^{2}}{\hat{\sigma}_{i}^{2}+\hat{\tau}_{2}}
$$

com distribuição $\theta_{\mathrm{i}} \mid \theta, \tau^{2} \stackrel{\text { indep. }}{\sim} \mathrm{N}\left(\theta, \tau^{2}\right)$ (Gaver et al.).

$\mathrm{O}$ valor de $\tau^{2}$ foi estimado pelo método da Máxima Verossimilhança Restrita (MVR), segundo Normand (1999), por:

$$
\hat{\tau}_{M V R}^{2}=\frac{\sum_{i=1}^{k} w_{i}^{2}(\hat{\tau})\left[\frac{k}{k-1}\left(Y_{i}-\hat{\theta}_{M V R}\right)^{2}-\operatorname{Var}\left(\hat{\theta}_{i}\right)\right]}{\sum_{i=1}^{k} W_{i}^{2}(\hat{\tau})}
$$

utilizando-se o procedimento MIXED (SAS, 1996).

Para se obter a estimativa combinada do coeficiente de correlação, aplicou-se a função inversa $r_{+}=\frac{\exp \left\{2 z_{+}\right\}-1}{\exp \left\{2 z_{+}\right\}+1}$ (Hedges \& Olkin, 1985).

A análise de subgrupo foi realizada com 0 objetivo de verificar a existência ou não de homogeneidade dentro e entre subgrupos específicos, adotando-se a estratificação por época de publicação do estudo. A homogeneidade em relação aos subgrupos foi testada sob as hipóteses, $H_{0}: \theta_{1+}=\theta_{2+}=\theta_{3+}$,

$$
\mathrm{H}_{1}:\left\{\begin{array}{l}
\theta_{11}=\ldots=\theta_{1 \mathrm{~m}_{1}}=\theta_{1+} \\
\theta_{21}=\ldots=\theta_{2 \mathrm{~m}_{2}}=\theta_{2+} \\
\theta_{31}=\ldots=\theta_{3 \mathrm{~m}_{3}}=\theta_{3+}
\end{array}\right.
$$

versus, $\mathrm{H}_{\mathrm{A}}$ : pelo menos um coeficiente de correlação transformado difere dos demais. Para testar a hipótese $\mathrm{H}_{0}$, que não existe variação entre os grupos de estudo, utilizou-se a estatística $Q_{\text {entre }}$, já descrita; e para testar a hipótese $\mathrm{H}_{1}$, que não existe variação dentro dos grupos de estudo, utilizou-se a estatística $Q_{\text {dentro }}=\sum_{i=1}^{k} \sum_{j=1}^{m_{i}} w_{i j}\left(\hat{\theta}_{i j}-\hat{\theta}_{i+}\right)^{2}$, a qual tem distribuição de qui-quadrado com k-p graus de liberdade, onde na análise de subgrupo $k=3$. $O$ teste de homogeneidade e as estimativas combinadas $\left(z_{+}, r_{+}\right)$ para cada subgrupo foram realizadas por macro e procedimentos específicos do SAS, propostos por Wang \& Bushman (1999).

A etapa final da meta-análise consistiu em retirar os estudos considerados "outliers", refazer todas as etapas, e avaliar o impacto daqueles estudos nas estimativas combinadas do coeficiente de correlação.

\section{RESULTADOS E DISCUSSÃO}

$\mathrm{Na}$ Tabela 1 estão relacionados em ordem cronológica todos os estudos com respectiva raça, tamanho da amostra (n) e coeficiente de correlação (r). Após aplicado o critério de inclusão, dos 39 estudos permaneceram 34. Para estes foram calculados o coeficiente de correlação transformado (z) e a variância de $z(1 /(n-3))$.

O "dot plot" para os dados está apresentado na Figura 1 contendo, o número do estudo que foi incluído na meta-análise (Estudo), os coeficientes de correlação genéticos não transformados (Efeito), os limites dos intervalos a $95 \%$ de confiança calculados para cada coeficiente de correlação (Inferior, Superior) e os maiores e menores valores encontrados para estes intervalos (mín., máx.). O intervalo de confiança para o estudo 27 indica uma correlação negativa, que o intervalo de confiança para o estudo 25 inclui o valor zero, que os estudos $17,21,25,27$ e 37 são considerados "outliers". Para os demais estudos, este dispositivo gráfico demonstra que existe uma correlação positiva entre peso ao nascimento e peso à desmama, com aparente heterogeneidade entre eles.

$O$ valor da estatística $Q_{\text {entre }}$ para os 34 estudos foi 40105 (com 33 graus de liberdade e "P-value" < $0,01)$, existindo, desta maneira, fortes evidências para rejeitar a hipótese da homogeneidade entre os valores de $z$.

Sob a suposição do modelo de efeitos fixos, utilizando a ponderação $w_{i}$, a estimativa combinada foi $r_{+} \cong 0,531$. Na aproximação através do modelo de efeito aleatório o componente de variância estimado entre os estudos foi $\hat{\tau}_{\mathrm{MVR}}^{2}=0,291$, e a estimativa combinada para o coeficiente de correlação foi $r_{+}^{*} \cong 0,576$. Os dois valores podem ser considerados altos de acordo com Cohen (1988). 
Tabela 1 - Relação cronológica dos estudos com as diferentes raças, número de observações ( $\mathrm{n}$ ), coeficiente de correlação $(r)$, coeficiente de correlação transformado $(z)$, variância de $z$, subgrupo e referência bibliográficas.

\begin{tabular}{|c|c|c|c|c|c|c|c|}
\hline Estudo & Raça & $\mathrm{n}$ & r & z & $1 /(n-3)$ & Sub & Referência \\
\hline 1 & Brahman & 1049 & 0,84 & 1,22117 & 0,00096 & 1 & Berruecos \& Robison (1968) \\
\hline $2^{b}$ & Nelore & 417 & 0,44 & - & - & 1 & Felício et al. (1976) \\
\hline $3^{b}$ & Guzerá & 399 & 0,26 & - & - & 1 & Felício et al. (1976) \\
\hline $4^{b}$ & Gir & 261 & 1,18 & - & - & 1 & Felício et al. (1976) \\
\hline 5 & Nelore & 1580 & 0,81 & 1,12703 & 0,00063 & 1 & Mortari (1976) \\
\hline $6^{b}$ & Gir & 193 & 0,21 & - & - & 1 & Torres et al. (1979) \\
\hline 7 & Nelore & 1356 & 0,67 & 0,81074 & 0,00074 & 1 & Pimenta-Filho (1980) \\
\hline 8 & Nelore & 12339 & 0,25 & 0,25541 & 0,00008 & 2 & Olive ira-Filho \& Duarte (1981) \\
\hline 9 & Nelore & 831 & 0,67 & 0,81074 & 0,00121 & 2 & Baroni (1982) \\
\hline 10 & Nelore & 998 & 0,40 & 0,42365 & 0,00101 & 2 & Silva (1983) \\
\hline 11 & Tabapuã & 1857 & 0,76 & 0,99622 & 0,00054 & 2 & Ledic (1983) \\
\hline 12 & Nelore & 1028 & 0,38 & 0,40006 & 0,00098 & 2 & Silva et al. (1983) \\
\hline 13 & Guzerá & 659 & 0,53 & 0,59015 & 0,00152 & 2 & Marques et al. (1983) \\
\hline 14 & Brahman & 2588 & 0,30 & 0,30952 & 0,00039 & 2 & Verde et al. (1983) \\
\hline 15 & Nelore & 2944 & 0,47 & 0,51007 & 0,00034 & 2 & Nobre et al. (1985) \\
\hline 16 & Nelore & 4249 & 0,19 & 0,19234 & 0,00024 & 2 & Milagres et al. (1985) \\
\hline 17 & Nelore & 2282 & 0,99 & 2,64665 & 0,00044 & 2 & Mariante \& Zancaner (1985) \\
\hline $18^{b}$ & Guzerá & 485 & 0,73 & - & - & 2 & Oliveira et al. (1986) \\
\hline 19 & Gudali & 31488 & 0,81 & 1,12703 & 0,00003 & 2 & Iloeje (1986) \\
\hline 20 & Nelore & 5701 & 0,40 & 0,42365 & 0,00018 & 2 & Rosa et al. (1986) \\
\hline 21 & Nelore & 4662 & 0,92 & 1,58903 & 0,00022 & 2 & Amaral (1986) \\
\hline 22 & Nkone & 1864 & 0,54 & 0,60416 & 0,00054 & 2 & Tawonezvi et al. (1986) \\
\hline 23 & Nelore & 5483 & 0,34 & 0,35409 & 0,00018 & 2 & Silva et al. (1987) \\
\hline 24 & Nelore & 2550 & 0,54 & 0,60416 & 0,00039 & 2 & Cardellino \& Castro (1987) \\
\hline 25 & Boran & 3959 & 0,01 & 0,01000 & 0,00025 & 2 & Arnason \& Kassa-Mersha (1987) \\
\hline 26 & Nelore & 590 & 0,16 & 0,16139 & 0,00170 & 2 & Scherré et al. (1988) \\
\hline 27 & Nelore & 574 & $-0,12$ & 0,12058 & 0,00175 & 2 & Scherré et al. (1988) \\
\hline 28 & Nelore & 18751 & 0,18 & 0,18198 & 0,00005 & 2 & Eler et al. (1989) \\
\hline 29 & Gobra & 1401 & 0,49 & 0,53606 & 0,00072 & 2 & Abassa et al. (1989) \\
\hline 30 & Guzerá & 585 & 0,45 & 0,48470 & 0,00172 & 2 & Martins-Filho \& Lôbo (1989) \\
\hline 31 & Nelore & 2604 & 0,28 & 0,28768 & 0,00039 & 3 & Ayala et al. (1991) \\
\hline 32 & Guzerá & 748 & 0,64 & 0,75817 & 0,00134 & 3 & Oliveira et al. (1993) \\
\hline 33 & Nelore & 27549 & 0,23 & 0,23419 & 0,00004 & 3 & Eler et al. (1994) \\
\hline 34 & Nelore & 18200 & 0,42 & 0,44769 & 0,00006 & 3 & Lôbo et al. (1995a) \\
\hline 35 & Nelore & 17131 & 0,42 & 0,44769 & 0,00006 & 3 & Reyes et al. (1995) \\
\hline 36 & Nelore & 3679 & 0,39 & 0,41180 & 0,00027 & 3 & Salles (1995) \\
\hline 37 & Nelore & 2567 & 0,91 & 1,52752 & 0,00039 & 3 & Souza \& Ramos (1995) \\
\hline 38 & Nelore & 31114 & 0,63 & 0,74142 & 0,00003 & 3 & Eler et al. (1996) \\
\hline 39 & Nelore & 688 & 0,83 & 1,18814 & 0,00146 & 3 & Martins et al. (2000) \\
\hline
\end{tabular}

aSubgrupo: "1" décadas de 60 e 70 , "2" década de 80 , "3" década de 90 . 'estudos retirados da meta-análise pelo critério de exclusão (n<500 observações).

Conforme o teste de homogeneidade existem diferenças substanciais entre os estudos. Assim, o modelo de efeito aleatório pode ser mais apropriado para as estimativas individuais de correlação genética dos diferentes estudos.

A amplitude dos intervalos de confiança para os modelos de efeito fixo e de efeito aleatório está representado na Figura 2. Os intervalos de confiança foram $0,527 \leq r_{+} \leq 0,535$ e $0,442 \leq r_{+}^{*} \leq 0,684$ para 0 modelo de efeitos fixos (MEF) e o modelo de efeitos aleatórios (MEA), respectivamente, indicando uma amplitude maior para o último. A análise de subgrupo foi realizada incorporando o componente de variância entre os estudos, ou seja, sob o modelo aleatório. Estão expostos na Tabela 2 os resultados da análise para os três subgrupos de interesse. Existem fortes evidências para rejeitar as hipóteses de nulidade, isto é, tanto entre como dentro dos subgrupos de estudos não existe homogeneidade para os coeficientes de correlação. 


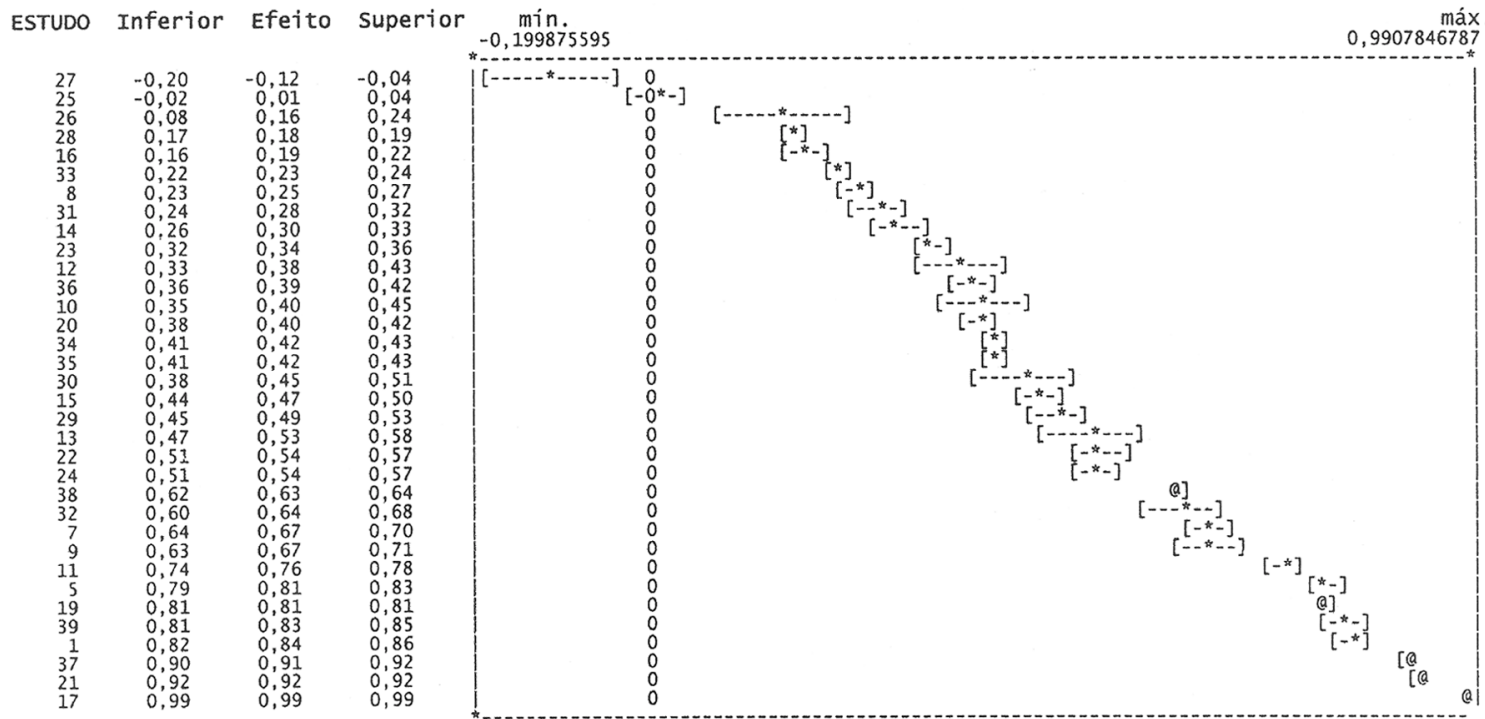

Figura 1 - "Dot-plot" para a correlação entre peso ao nascimento e peso à desmama.

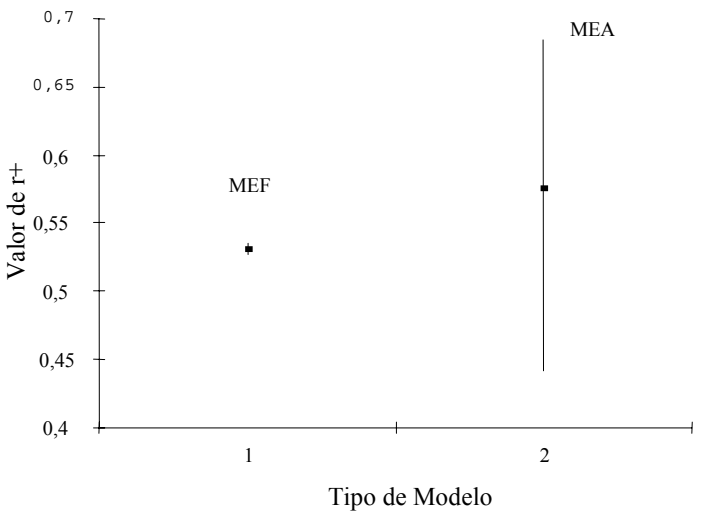

Figura 2 - Intervalos de Confiança do Modelo de Efeito fixo (MEF) e Modelo de Efeito Aleatório (MEA).

Tabela 2 - Análise da heterogeneidade para os subgrupos de estudo.

\begin{tabular}{lrcc}
\hline Causa de Heterogeneidade & GL & ESTAT.-Q & "P-value" \\
\hline Entre Subgrupos & 2 & 2006,55 & 0,000 \\
Dentro Subgrupos & 31 & 38098,2 & 0,000 \\
Dentro(Anos 70) & 2 & 117,321 & 0,000 \\
Dentro(Anos 80) & 21 & 30903,3 & 0,000 \\
Dentro(Anos 90) & 8 & 7077,51 & 0,000 \\
Total & 33 & 40104,7 & 0,000 \\
\hline
\end{tabular}

As estimativas combinadas para os coeficientes de correlação referentes aos subgrupos de estudos estão apresentadas na Tabela 3 , juntamente com os respectivos intervalos de confiança. O valor da estimativa combinada para a década de 70 pode ser considerado muito alto, e a amplitude do seu intervalo de confiança grande. Tal fato pode ter ocorrido em virtude deste subgrupo envolver estudos mais antigos, indicando, provavelmente, uma qualidade inferior dos dados quando comparados aqueles das outras duas décadas, e/ou pelo baixo número de estudos contidos neste subgrupo.
Tabela 3 - Estimativas combinadas para os subgrupos e intervalos de confiança.

\begin{tabular}{lccc}
\hline Subgrupo & $\mathrm{r}_{+}^{*}$ & Limite Infe rior & Limite Superior \\
\hline Década 70 & 0,783 & 0,415 & 0,931 \\
Década 80 & 0,534 & 0,354 & 0,675 \\
Década 90 & 0,586 & 0,308 & 0,771 \\
Total & 0,576 & 0,442 & 0,684 \\
\hline
\end{tabular}

Os valores das estimativas combinadas para as décadas de 80 e 90 podem também ser considerados altos. As amplitudes dos seus intervalos de confiança são menores daquelas da década de 70 , fato que pode indicar uma melhor qualidade dos estudos dessas duas últimas décadas em decorrência de uma possível melhoria dos métodos de estimação dos parâmetros genéticos e da qualidade e quantidade dos banco de dados envolvidos.

A análise de sensibilidade foi realizada, com o intuito de verificar se o resultado combinado foi afetado, ou não, pelos estudos considerados "outiliers" potenciais. Como observado anteriormente por meio do "dot plot" (Figura 1), os estudos que podem ser considerados "outliers" são os de número 17, 21, 25, 27 e 37, os quais foram eliminados. Na nova análise o valor da estatística $Q_{\text {entre }}$ foi 21565 (com 28 graus de liberdade e "P-value" $<0,01$ ), caracterizando a não homogeneidade entre os mesmos. Para o modelo aleatório, o componente de variância estimado foi $\hat{\tau}_{\mathrm{MVR}}^{2}=0,099$ e a estimativa combinada obtida para os 29 estudos $\left(r_{+}^{*}=0,518\right)$ foi inferior àquela obtida para os 34 estudos.

A retirada dos cinco estudos determinou uma redução importante no valor de $\hat{\tau}_{\mathrm{MVR}}^{2}$ proporcionando uma sensível redução no intervalo de confiança da estimativa combinada, pelo modelo de efeito aleatório $\left(0,429 \leq r_{+}^{*} \leq 0,597\right)$ relativamente aos valores obtidos na análise anterior. 
Tabela 4 - Análise da heterogeneidade para os subgrupos de estudo na análise de sensibilidade.

\begin{tabular}{lrcc}
\hline Causa de Heterogeneidade & GL & ESTAT.-Q & "P-value" \\
\hline Entre Subgrupos & 2 & 1639,86 & 0,000 \\
Dentro Subgrupos & 26 & 19925,0 & 0,000 \\
Dentro(Anos 70) & 2 & 117,321 & 0,000 \\
Dentro(Anos 80) & 17 & 15466,2 & 0,000 \\
Dentro(Anos 90) & 7 & 4341,54 & 0,000 \\
Total & 28 & 21564,9 & 0,000 \\
\hline
\end{tabular}

Tabela 5 - Estimativas combinadas para os subgrupos e intervalos de confiança, para a análise de sensibilidade.

\begin{tabular}{lccc}
\hline Subgrupo & & Limite Inferior & Limite Superior \\
\hline Déca da 70 & 0,783 & 0,601 & 0,888 \\
Déca da 80 & 0,461 & 0,338 & 0,568 \\
Déca da 90 & 0,510 & 0,332 & 0,654 \\
Total & 0,518 & 0,429 & 0,597 \\
\hline
\end{tabular}

Foi realizada a análise de subgrupo, indicando não existir homogeneidade entre e dentro dos subgrupos, sugerindo novamente o uso da aproximação de efeito aleatório para modelagem de variação desses dados (Tabela 4).

A eliminação dos estudos considerados "outliers" acarretou numa redução importante nas estimativas combinadas dos coeficientes de correlação genética, assim como na amplitude de seus intervalos de confiança (Tabela 5) comparativamente aos resultados obtidos para os valores dos 34 estudos (Tabela 3).

\section{CONCLUSÕES}

A confiabilidade da meta-análise reside tanto na aplicação de técnicas estatísticas apropriadas para sintetizar a informação de estudos distintos, quanto na quantidade e sobretudo qualidade dos mesmos.

A modelagem da variação entre os diferentes estudos é uma etapa importante na condução de uma meta-análise. Para a situação específica deste trabalho, observou-se que o modelo fixo não foi apropriado em virtude da falta de homogeneidade das estimativas de correlação genética. O modelo aleatório foi mais eficiente. A formação de subgrupos permite modelar mais eficientemente a variação entre os estudos distintos.

O uso de técnicas de meta-análise para sintetizar resultados de revisão de parâmetros genéticos é recomendável.

\section{REFERÊNCIAS BIBLIOGRÁFICAS}

COHEN, J. Statistical power analysis for the behavioral sciences. 2.ed. New York: Academic Press, 1988. 567p.

COOPER, H.M. Integrating research: a guide for literature reviews. 2.ed. Newbury Park: Sage, 1990. 157p.

COSTA, P.A.B. Um enfoque segundo a teoria de conjuntos difusos para a meta-análise. Florianópolis, 1999. 153p. Tese (Doutorado) - Universidade Federal de Santa Catarina.

DerSIMONIAN, R.; LAIRD, N. Meta-analysis in clinical trials. Controlled Clinical Trials, v.7, p.177-188, 1986.

EGGER, M.; SMITH, G.D. Meta-analysis: potentials and promise. British Journal of Medicine, v.315, p.1371-1374, 1997.

EGGER, M.; SMITH, G.D.; PHILLIPS, A.N. Meta-analysis: principles and procedures. British Journal of Medicine, v.315, p.1533-1537, 1997.

ELER, J.P.; FERRAZ, J.B.S.; SILVA, P.R. Estimação simultânea de parâmetros genéticos para características de importância econômica na raça nelore, com a utilização de modelos animais. In: REUNIÃO ANUAL DA SOCIEDADE BRASILEIRA DE ZOOTECNIA, 33., Fortaleza, 1996. Anais. Viçosa: SBZ, 1996.

FAGARD, R.H.; STAESSEN, J.A.; THIJS, L. Advantages and disadvantages of the meta-analysis approach. Journal of Hypertension, v.14, p.9-13, 1996. Supplement 2

GAVER, J.R.; DRAPER, D.P.; GOEL, P.K.; GREENHOUSE, J.B.; HEDGES, L.V.; MORRIS, C.N.; WATERNAUX, C. Panel on statistical issues and opportunities for research in the combination of information. Washington: National Academic Press, 1992. 217p.

GLASS, G.V. Primary, secundary, and meta-analysis of research. Educational Researcher, v.6, p.3-8, 1976.

HEDGES, L.V.; OLKIN, I. Statistical methods for meta-analysis. London: Academic Press, 1985. 369p.

HOUWELINGEN, H.C.V. The future of biostatistics: expecting the unexpected. Statistics in Medicine, v.16, p.2773-2784, 1997.

KIRBY, K.N. Advanced data analysis with SYSTAT. New York: Van Nostrand Reinhold, 1993. 475p.

KOOTS, K.R.; GIBSON, J.P.; SMITH, C.; WILTON, J.W. Analyses of publised genetic parameter estimates for beef production traits: 1. Heritability. Animal Breeding Abstracts, v.62, p.309-338, 1994a.

KOOTS, K.R.; GIBSON, J.P.; SMITH, C.; WILTON, J.W. Analyses of publised genetic parameter estimates for beef production traits: 2. Phenotypic and genetic correlations. Animal Breeding Abstracts, v.62, p.826-853, $1994 \mathrm{~b}$.

LÔBO, R.N.B; MADALENA, F.E.; VIEIRA, A.R. Average estimates of genetic parameters for beef and dairy cattle in Tropical regions. Animal Breeding Abstracts, v.68, p.433-462, 2000.

MARTINS, G.A.; MARTINS FILHO, R.; LIMA, F.A.M.; LÔBO, R.N.B. Influência dos fatores genéticos e de meio sobre o crescimento de bovinos da raça Nelore no estado do Maranhão. Revista Brasileira de Zootecnia, v.29, p.103-107, 2000.

MERCADANTE, M.E.Z.; LÔBO, R.B.; REYES, A. Parámetros genéticos para características de crescimiento en cebuínos de carne. Archivo Latinoamericano Producción Animal, v.3, p.45-89, 1995.

MOHIUDDIN, G. Estimates of genetic and phenotypic parameters of some trais in beef cattle. Animal Breeding Abstracts, v.61, p.495-522, 1993.

NORMAND, S.T. Tutorial in biostatistics meta-analysis: formulating, evaluating, combining, and reporting. Statistics in Medicine, v.18, p.321-359, 1999.

SAS INSTITUTE. SAS procedures guide. Version 6. 3.ed. Cary: Statistical Analysis System Institute, 1990a.

SAS INSTITUTE. SAS/STAT user's guide. Version 6. 4.ed. Cary: Statistical Analysis System Institute, 1990b. 846p.

SAS INSTITUTE. SAS/STAT software: changes and enhancements through release 6.07. 6.12. Cary: Statistical Analysis System Institute, 1996.

WANG, M. C.; BUSHMAN, B. J. Integration results: through meta-analytic review using SAS software. Cary: Statistical Analysis System Institute, 1999. 400p.

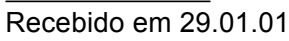

